\title{
Focusing On Relativistic universe and Cosmic Evolution: the FORCE mission
}

\author{
Koji Mori*1, Takeshi Go Tsuru ${ }^{2}$, Kazuhiro Nakazawa ${ }^{3}$, Yoshihiro Ueda ${ }^{4}$, Takashi \\ Okajima $^{5}$, Hiroshi Murakami ${ }^{6}$, Hisamitsu Awaki ${ }^{7}$, Hironori Matsumoto ${ }^{8}$, Yasushi \\ Fukazawa $^{9}$, Hiroshi Tsunemi ${ }^{8}$, Tadayuki Takahashi ${ }^{10}$, and William W. Zhang ${ }^{5}$ \\ ${ }^{1}$ Department of Applied Physics and Electronic Engineering, University of Miyazaki, Miyazaki \\ 889-2192, Japan; E-mail: morieastro.mivazaki-u.ac.70; ; ${ }^{2}$ Department of Physics, \\ Kyoto University, Kyoto 606-8502, Japan; ${ }^{3}$ Department of Physics, University of Tokyo, Tokyo \\ 113-0033, Japan; ${ }^{4}$ Department of Astronomy, Kyoto University, Kyoto 606-8502, Japan; \\ ${ }^{5}$ NASA/Goddard Space Flight Center, MD 20771, USA; ${ }^{6}$ Department of Information Science, \\ Faculty of Liberal Arts, Tohoku Gakuin University, Miyagi 981-3193, Japan; ${ }^{7}$ Department of \\ Physics, Ehime University, Ehime 790-8577, Japan; ${ }^{8}$ Department of Earth and Space Science, \\ Osaka University, Osaka 560-0043, Japan; ${ }^{9}$ Department of Physical Science, Hiroshima \\ University, Hiroshima 739-8526, Japan; ${ }^{10}$ Institute of Space and Astronautical Science (ISAS), \\ Japan Aerospace Exploration Agency (JAXA), Kanagawa 252-5210, Japan
}

The Focusing On Relativistic universe and Cosmic Evolution (FORCE) mission is proposed as a future Japan-lead X-ray observatory to be launched in the mid 2020s. FORCE is a successor of Hitomi, focusing on the braodband X-ray imaging spectroscopy in $1-80 \mathrm{keV}$ with a significantly higher angular resolution of $<15^{\prime \prime}$ in half-power diameter. The sensitivity above $10 \mathrm{keV}$ will be 10 times higher than that of any previous hard X-ray missions with simultaneous soft X-ray coverage. The satellite is planned to be launched by the Epsilon vehicle by ISAS/JAXA. In the current design concept, FORCE is equipped with three identical pairs of supermirrors and wideband X-ray detectors. The focal length of the mirrors is $10 \mathrm{~m}$. The silicon mirror with multi-layer coating is our primary choice of optics to achieve a good angular resolution for the wide energy band while maintaining a light weight. The detector is a hybrid of a SOI-CMOS silicon-pixel detector and a CdTe detector responsible for the softer and harder energy bands, respectively. It is basically a descendant of the hard X-ray imager onboard Hitomi with its soft-band detector replaced with the SOI-CMOS. The primary scientific objective of the FORCE mission is to trace the cosmic formation history by searching for "missing black holes" in the entire range of the mass spectrum of BHs: "buried" supermassive black holes (SMBHs) $\left(>10^{4} M_{\odot}\right)$, intermediate-mass black holes $\left(10^{2}-10^{4} M_{\odot}\right)$, and "orphan" stellar-mass black holes $\left(<10^{2} M_{\odot}\right)$. Also, investigation of the nature of relativistic particles at various astrophysical shocks is in our scope, with highangular-resolution X-ray observations with the broadband coverage. FORCE will open a new era in these fields.

XII Multifrequency Behaviour of High Energy Cosmic Sources Workshop 12-17 June, 2017

Palermo, Italy

* Speaker. 


\section{INTRODUCTION}

The hard X-ray imaging spectroscopy is a highly sensitive probe of non-thermal emission, such as synchrotron radiation, Compton scattering, and nuclear $\gamma$-ray lines, from high-energy objects. A "supermirror" technology expands its ability by focusing hard X-rays above $10 \mathrm{keV}$ beyond the conventional softer energy band. NuSTAR is the first hard-X-ray-dedicated satellite to utilize the supermirror optics[四], and Hitomi, formerly called ASTRO-H, has followed[[]]. Whereas this new probe in the hard X-ray band alone is very powerful, simultaneous coverage on the soft X-ray band would be ideal, considering the broadband and time-variable nature of non-thermal emission. It would be especially beneficial in observing the sources in which multiple emission components exists. Indeed, many NuSTAR and XMM-Newton/Suzaku joint observations have demonstrated the benefit of the simultaneous broadband coverage[B]. Hitomi realized the broadband coverage, carrying independent, soft- and hard-X-ray-imaging systems. However, if a single focal plane detector were sensitive for a broad X-ray band, a combination of a supermirror and such a "superdetector" would be the most effective way of achieving the objective with limited resources, because suppermirrors focus not only hard X-rays but also soft X-rays.

Here we present an overview of the Focusing On Relativistic universe and Cosmic Evolution (FORCE) mission, including the design of the satellite and the scientific objectives. FORCE is an ISAS/JAXA medium-class mission proposed to be launched in the mid 2020s. FORCE provides a capability of the broadband X-ray imaging spectroscopy in 1-80 keV with an angular resolution of $<15^{\prime \prime}$ in half-power diameter (HPD), and achieves a sensitivity above $10 \mathrm{keV}$ that is 10 times higher than any previous missions with simultaneous soft X-ray coverage.

\section{SCIENTIFIC OBJECTIVES AND REQUIREMENTS}

Our primary scientific objective is to trace the cosmic formation history by searching for "missing black holes" in the entire range of the mass spectrum of black holes. The population of missing black holes includes "buried" supermassive black holes (SMBHs) $\left(>10^{4} M_{\odot}\right)$, which reside in centers of galaxies in a cosmological distance, intermediate-mass black holes $\left(10^{2}-10^{4} M_{\odot}\right)$, which are possible seeds from which SMBHs grow, and "orphan" stellar-mass black holes $\left(<10^{2} M_{\odot}\right)$, which have no companion, in our Galaxy. In addition to these missing BHs, investigation of the nature of relativistic particles at various astrophysical shocks is also in our scope. In the following section, we will briefly review the SMBHs case, which is our primary objective.

\subsection{Supermassive Black Hole: Co-evolution with their host galaxies}

One of the key questions with the evolution of active galactic nuclei (AGNs) is how many buried and heavily obscured (Compton-thick) AGNs exist in the universe, as well as the extent of their contribution to the growth of SMBHs. It still remains unclear whether or not Compton-thick and Compton-thin AGNs are intrinsically the same population. Theories predict that galaxies may contain Compton-thick AGNs shortly after major mergers, which are surrounded by a huge amount of dust produced by the merger-driven starburst activity [困, []. These Compton-thick AGNs may be in the rapidly growing phase of the SMBH, and hence are key objects to understand the mechanism of the galaxy-SMBH co-evolution. 
Most of present X-ray surveys were performed in energy bands below $10 \mathrm{keV}$, in which the primary emission component from Compton-thick AGNs is hardly detectable. Although recent all-sky hard X-ray surveys above $\sim 10 \mathrm{keV}$ have started to find a part of this population in the local universe, the cosmological evolution of their X-ray luminosity function (XLF) is still virtually unknown. The sole direct way to unveil the cosmological evolution of Compton-thick AGNs and their contribution to the hard X-ray background (XRB) is to perform hard X-ray surveys in the energy band above $10 \mathrm{keV}$ with various depths and widths, as has been done in the past $\sim 50$ years at energies below $10 \mathrm{keV}$.

Recent imaging surveys by NuSTAR with an arc-minute angular resolution have resolved $\sim 35 \%$ of the hard XRB in the $8-24 \mathrm{keV}$ band[G], providing us with new insights on Comptonthick AGN populations. However, $\sim 35 \%$ is hardly sufficient to solve the origin of the hard XRB. The next logical step is to increase the sensitivity; for example, with a $>10$ times higher sensitivity than those by NuSTAR, $\sim 80 \%$ of the hard XRB is estimated to be resolved into individual AGNs. The sensitivity required is $2-3 \times 10^{-15} \mathrm{erg} \mathrm{cm}^{-2} \mathrm{~s}^{-1}$ in the $10-40 \mathrm{keV}$ band. The population synthesis model by Ueda et al. (2014)[ [ $]$ ] predicts that the fraction of Compton-thick AGNs drastically increases around this flux limit. With this sensitivity, it would be also feasible to give some significant constrains to the XLF of Compton-thick AGNs with $L_{\mathrm{X}}>10^{42} \mathrm{erg} \mathrm{s}^{-1} \mathrm{~cm}^{-2}$ by covering the redshift range corresponding to the number-density peak inferred from the XLF of Compton-thin AGNs.

\subsection{Scientific requirements}

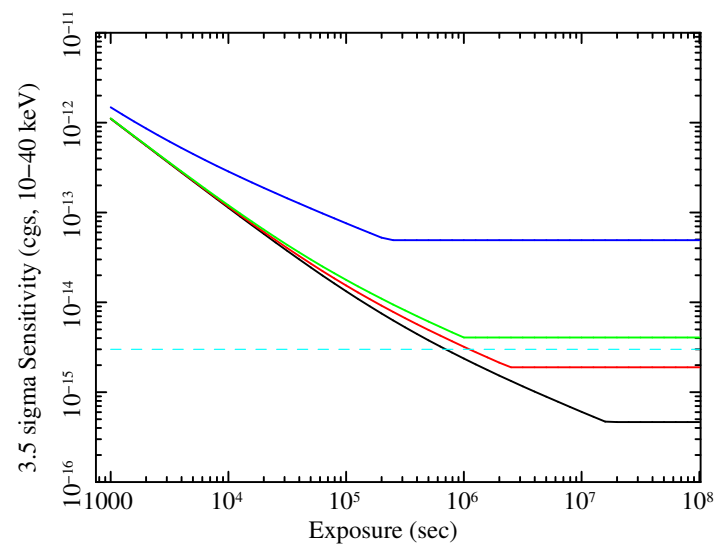

Figure 1: Sensitivity curves for deep surveys as a function of exposure time with different angular resolutions. Black, red, and green lines indicate the cases of HPDs of $10^{\prime \prime}, 15^{\prime \prime}$, and $20^{\prime \prime}$, respectively, while the blue line shows the Hitomi case. The cyan dashed line indicates the sensitivity required from the AGN survey (see text)

In order to achieve the aforementioned scientific objectives, X-ray observations with simultaneous broadband coverage and with, crucially, a high sensitivity in the hard X-ray band are required. The sensitivity to point sources is generally limited by exposure time, background, and 
source confusion. Figure $\mathbb{W}$ shows sensitivity curves for deep surveys as a function of exposure time for four different angular-resolution cases, including the Hitomi case, where the background level and effective area are assumed to be comparable to those of Hitomi hard X-ray telescope and detector combination, and the standard XRB model[ $[\mathrm{W}]$ is used. Although the sensitivity of an observation improves with a longer exposure time in general, there is a limit for the improvement due to source confusion limit. Thus, a higher angular resolution yields a better limiting sensitivity. In particular, the angular resolution of $<15^{\prime \prime}$ in HPD is required to achieve a limiting sensitively of $2-3 \times 10^{-15} \mathrm{erg} \mathrm{cm}^{-2} \mathrm{~s}^{-1}$, which is the requirement from the AGN survey described in the previous section.

\section{MISSION DESIGN AND SCIENCE INSTRUMENTS}

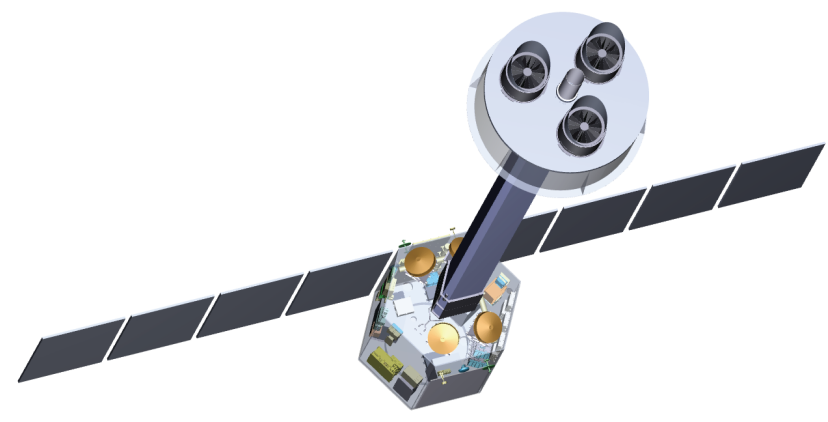

Figure 2: Schematic view of the FORCE satellite.

FORCE has a weight of about 1 metric ton, and is planned to be launched with the Epsilon vehicle, which is an ISAS/JAXA solid-fuel rocket. The satellite will be put into a circular orbit with an altitude of 500-600 km and inclination angle of 31 degrees or smaller which is similar to those of the past Japanese X-ray satellites. Figure $\square$ shows a schematic view of the satellite. FORCE carries three co-aligned identical pairs of a supermirror with a high angular resolution and a focal-plane detector with a broadband response. The supermirror and the detector are separated by a focal length of $10 \mathrm{~m}$; the long focal length is necessary to ensure a sufficient effective area for hard X-ray focusing. An extendable optical bench (EOB) is an engineering requirement for it, which can be stowed to fit inside the payload fairing during the launch and be deployed on-orbit. In the current design, the $10 \mathrm{~m}$ length is achieved by a combination of $2 \mathrm{~m}$ fixed optical bench (FOB) and $8 \mathrm{~m} \mathrm{EOB}$, and the mirror module is placed at the end of the EOB.

Table W summarizes key instrument parameters at the time of writing. High angular resolution of $<15^{\prime \prime}$ in the broadband of $1-80 \mathrm{keV}$ characterizes this mission. These parameters are defined by the design concepts of the mirrors and detectors, which are described in the following sections. 
Table 1: Instrument parameters

\begin{tabular}{|l|l|}
\hline Angular resolution (HPD) & $<15^{\prime \prime}$ \\
\hline Multi-layer Coating & $\mathrm{Pt} / \mathrm{C}$ \\
\hline Field of view (50\% response) at $30 \mathrm{keV}$ & $\sim 7^{\prime} \times 7^{\prime}$ \\
\hline Effective Area at $30 \mathrm{keV}$ & $370 \mathrm{~cm}^{2}$ \\
\hline Energy range & $1-80 \mathrm{keV}$ \\
\hline Energy resolution (FWHM) at $6 \mathrm{keV}$ & $<300 \mathrm{eV}$ \\
\hline Background & comparable to those of Hitomi HXI \\
\hline Timing resolution & several $\times 10 \mu \mathrm{s}$ \\
\hline Working temperature & $-20 \pm 1^{\circ} \mathrm{C}$ \\
\hline
\end{tabular}

\subsection{X-ray supermirror based on light-weight, high-resolution silicon mirror}

The mirror substrates of our X-ray supermirrors are made based on the single-crystal silicon mirror technology, which has a high potential for making light-weight and high-angular resolution X-ray optics[]. In general, a higher angular resolution is accompanied with a larger mass and higher production cost, which may well be one of the biggest barriers in any satellite-based missions with optics. The silicon mirror can provide us with a good compromise for this issue. Although a number of technical issues still remain to be verified experimentally before a set of flightready telescopes is manufactured, the silicon mirror with multi-layer coating is most promising and is our primary choice to achieve light-weight, affordable, and high-angular resolution optics.

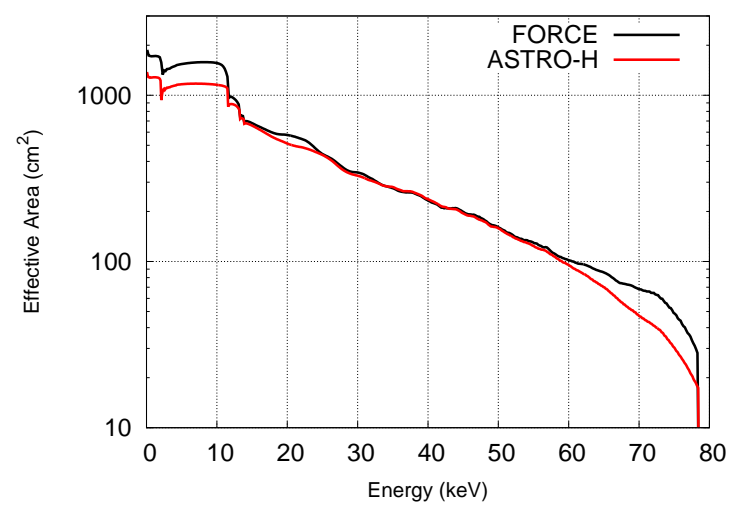

Figure 3: Total effective area of the three FORCE mirror modules in comparison with that of the Hitomi hard X-ray telescopes.

Figure 3 presents the total effective area of the three FORCE mirror modules based on the design in comparison with that of the Hitomi hard X-ray telescope (HXT). A throughput of 0.8, a reduction factor of the effective area from the design value, is assumed in this calculation. The total effective areas of FORCE and Hitomi HXT are more or less comparable above $10 \mathrm{keV}$; three mirror modules in FORCE against two in the Hitomi HXT compensate the $2 \mathrm{~m}$ shorter focal length of FORCE. FORCE has a larger effective area in the soft X-ray band due to its larger geometrical area. 


\subsection{Wideband hybrid X-ray imager}
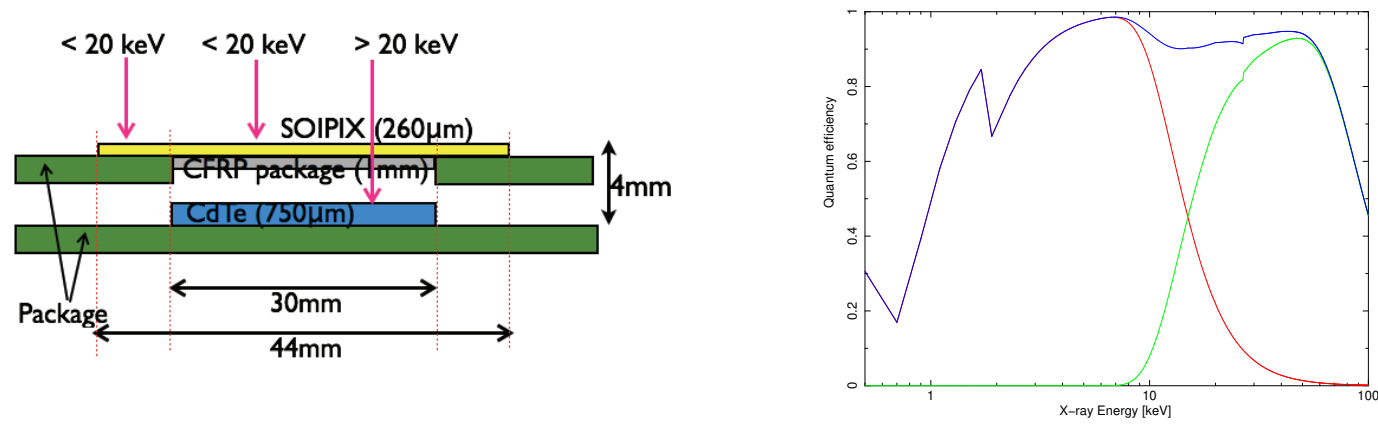

Figure 4: (Left) A schematic drawing of wideband hybrid X-ray imager (WHXI). (Right) Quantum efficiency of WHXI (blue). Contributions of SOIPIX (red) and CdTe (green) are also shown.

The focal-plane detector of FORCE, wideband hybrid X-ray imager (WHXI), has the same design concept as the hard X-ray imager (HXI) onboard Hitomi, comprising a Si and CdTe hybrid detector with an active shield. Figure $\mathrm{Q}$ left shows a schematic view of the WHXI focal plane part. The major difference between the HXI and WHXI is that whereas the HXI consists of four layers of double-sided Si strip detectors (DSSDs) and a single layer of CdTe double-sided strip (DSD) detector[Q], the WHXI replaces the former with a single SOI-CMOS pixel detector (SOIPIX) [प्,, []]. A low readout noise achievable by a SOIPIX is expected to lower the low-energy threshold down to $1 \mathrm{keV}$ and to provide a broadband energy response required for the focal-plane detector of FORCE. We should note that the CCD, which is the most standard and well-established soft$\mathrm{X}$-ray detector for imaging spectroscopy at the time of writing, is unsuitable in our case, where it must be physically placed next to the CdTe DSD (Fig. 团), because the operating temperature of the $\mathrm{CCD}$, typically $<-60^{\circ} \mathrm{C}$, is too compared with that of the CdTe of $\sim-20^{\circ} \mathrm{C}$. The SOIPIX has a good time resolution, and importantly, has a self-trigger function, which renders an anticoincidence technique, employable to achieve a low background. The mirror vignetting function has an energy dependence and provides a larger field of view in the lower energy band. Thus, the SOIPIX is designed to cover a larger area than CdTe DSD (Fig. 团). Figure 田 right shows the quantum efficiency (QE) of the WHXI. The QE at the lower energy side is currently limited by a dead layer of $1 \mu \mathrm{m}$ thickness at the surface of the SOIPIX. The active shield of the WHXI will be made with the same design concept as the one for the Hitomi HXI, which was proved to show a very low background in orbit[[]]. An upgrade to make it more simpler and easier to assemble may be considered.

\section{SUMMARY}

We have presented the design concept of future Japan-led X-ray mission, FORCE. The mission is characterized by broadband $(1-80 \mathrm{keV}) \mathrm{X}$-ray imaging spectroscopy with a high angular resolution $\left(<15^{\prime \prime}\right.$ in HPD), providing about 10 times higher sensitivity than the previous missions above $10 \mathrm{keV}$. FORCE will trace the cosmic formation history by searching the missing black holes in a wide mass range, and help us investigate the nature of relativistic particles at various astrophysical 
shocks. We are proposing FORCE to be launched to the orbit in the mid 2020s. FORCE will also be a hard X-ray counterpart of large soft-X-ray missions, such as ATHENA and Lynx, which are planned to be operated in the early 2030s.

\section{References}

[1] F. A. Harrison, W. W. Craig, F. E. Christensen, C. J. Hailey, W. W. Zhang, S. E. Boggs et al., The Nuclear Spectroscopic Telescope Array (NuSTAR) High-energy X-Ray Mission, Astrophyical Journal 780 (2014) 78.

[2] T. Takahashi, M. Kokubun, K. Mitsuda, R. L. Kelley and T. Ohashi, The ASTRO-H x-ray astronomy satellite, in Astronomical Telescopes and Instruments 2016 Proceeding, Proc. SPIE, 2016.

[3] G. Risaliti, F. A. Harrison, K. K. Madsen, D. J. Walton, S. E. Boggs, F. E. Christensen et al., A rapidly spinning supermassive black hole at the centre of NGC 1365, Nature 494 (2013) 449-451.

[4] D. B. Sanders, B. T. Soifer, J. H. Elias, B. F. Madore, K. Matthews, G. Neugebauer et al., Ultraluminous infrared galaxies and the origin of quasars, Astrophyical Journal 325 (1988) 74-91.

[5] P. F. Hopkins, L. Hernquist, T. J. Cox, B. Robertson and V. Springel, Determining the Properties and Evolution of Red Galaxies from the Quasar Luminosity Function, Astrophysical Journal Supplement 163 (2006) 50-79.

[6] F. A. Harrison, J. Aird, F. Civano, G. Lansbury, J. R. Mullaney, D. R. Ballantyne et al., The NuSTAR Extragalactic Surveys: The Number Counts of Active Galactic Nuclei and the Resolved Fraction of the Cosmic X-Ray Background, Astrophyical Journal 831 (2016) 185.

[7] Y. Ueda, M. Akiyama, G. Hasinger, T. Miyaji and M. G. Watson, Toward the Standard Population Synthesis Model of the X-Ray Background: Evolution of X-Ray Luminosity and Absorption Functions of Active Galactic Nuclei Including Compton -thick Populations, Astrophysical Journal 786 (2014) .

[8] W. W. Zhang, Lightweight and High-Resolution Single Crystal Silicon Optics for X-ray Astronomy, in Astronomical Telescopes and Instruments 2016 Proceeding, Proc. SPIE, 2016.

[9] K. Nakazawa, G. Sato, M. Kokubun, T. Enoto, Y. Fukazawa, K. Hagino et al., The hard $x$-ray imager (HXI) onboard ASTRO-H, in Astronomical Telescopes and Instruments 2016 Proceeding, Proc. SPIE, 2016.

[10] T. G. Tsuru, H. Matsumura, A. Takeda, T. Tanaka, S. Nakashima, Y. Arai et al., Development and performance of Kyoto's $x$-ray astronomical SOI pixel (SOIPIX) sensor, in Space Telescopes and Instrumentation 2014: Ultraviolet to Gamma Ray, Proc. SPIE, 2014.

[11] T. G. Tsuru, M. Itou, S. Ohmura, H. Matsumura, A. Takeda, H. Uchida et al., Spectral performances of Kyoto' $s x$-ray astronomical SOI pixel sensor in the frame and event-driven readout modes, in Astronomical Telescopes and Instruments 2016 Proceeding, Proc. SPIE, 2016.

\section{DISCUSSION}

YURI EVANGELISTA: What is the status and the performance of the CMOS detector? How thick is the depletion layer? 
KOJI MORI: Our CMOS detector is already properly working in the event-driven mode. We are currently working on an improvement of the energy resolution in this mode. That in the framereadout mode meets the mission requirement. The thickness of the depletion layer reaches up to $500 \mu \mathrm{m}$. 